\title{
Gender-Specific Aspects in Gastrointestinal Medicine and Surgery
}

\author{
Discussion Leader: Beate Rau (Berlin) \\ Participants: \\ Martin Angele (Munich) Santiago Gonzalez-Moreno (Madrid) \\ Georg Kähler (Mannheim) Corinna Langelotz (Berlin) \\ Gabriela Möslein (Bochum) Sabine Oertelt-Prigione (Berlin) \\ Andrea Riphaus (Laatzen) Ute Seeland (Berlin)
}

\begin{abstract}
Question 1: What does the term 'gender medicine' mean to you, and to which extent do you apply a gender-specific approach in your clinical practice, i.e. during medical consultation?
\end{abstract}

Angele: Several physiological and pathophysiological, pharmacological, social, and psychological differences between male and female patients have been identified with potential impact for treatment strategies. The term 'gender medicine' is a result of the awareness of those gender differences and applies to all fields of medicine. The recent importance of gender and associated differences in medicine will open new fields with potential implications for the clinical arena. At present, gender medicine is in a phase of finding and documenting gender-specific differences. The next step is to develop novel therapeutic approaches for the clinical arena, thereby considering gender and hormonal status. For me as an oncological surgeon, gender does not play a pivotal role for decisions regarding the treatment of our patients at the moment although anatomical differences exist.

Gonzalez-Moreno: I must admit that I am not familiar with the term 'gender medicine'. I gather it has to do with the differences in medical management or outcomes of individuals due to their difference in gender. My participation in this interdisciplinary discussion makes me aware of this topic for the first time, showing that maybe it should be more present in my (our) daily clinical activity.

Having carefully thought about it, and focusing on gender-non-specific diseases or disease sites (excluding breast or ovarian disease in women or prostate in men), I do not think that I have a different approach to my patients based on their gender during medical consultation. I treat all kinds of different patients that need a different approach based on their background, way to confront disease, social and family support, and sensibility. These are the factors that I consider to have an influence on the way I approach patients, rather than their gender. However, I would certainly be open to modulating my approach if I find new suggestions in this discussion.

Kähler: We try to integrate gender-specific problems into our daily clinical routine. For example, we know that women have an increased risk for post-ERCP (endoscopic retrograde cholangiopancreatography) pancreatitis. Therefore, they receive a prophylaxis with indometacine.

Question 2: According to your experience, who does profit most from preventive colonoscopy: men or women? Why?

Riphaus: Although some studies have shown that men are at a greater age-specific risk for the development of advanced colorectal neoplasia than women, the recommended age for referring patients to screening colonoscopy is still independent of sex and recommended at the age of 50 years. Therefore, data reflecting the gender-specific rate of adenoma detection in men and women are necessary. A cohort study encompassing 44,350 participants in a national screening colonoscopy program over a 4-year period (2007-2010) in Austria could show that the prevalence of advanced ade-

\section{KARGER}

Fax +497614520714

Information@Karger.com

www.karger.com (c) 2014 S. Karger GmbH, Freiburg

Accessible online at:

www.karger.com/vim 
nomas in 50- to 54-year-old individuals was 5.0\% in men but $2.9 \%$ in women. The number needed to screen for adenomas in men was 20 compared to 34 in women. The result could not show a statistical significance between the prevalence and number needed to screen for advanced adenomas in men aged between 45 and 49 years compared with women aged between 55 and 59 years (3.8 vs. $3.9 \%$ and 26.1 vs. $26 \%$, respectively). Comparable data are given for current large cohort studies in Germany. Therefore, current guideline developments should address this gender-specific aspect with subsequent sex-related recommendations for the first surveillance colonoscopy.

Kähler: Our experience is that men have sporadic adenocarcinoma in the colorectum earlier. Therefore, a correction of the guidelines would be necessary.

\section{Question 3: Which field is the most interesting and relevant when focusing on gender-related differences in the treatment of patients?}

Angele: At the moment, we are at the beginning of understanding the importance of gender differences in the treatment of our patients. In this respect, both gender and hormonal differences should be considered and will certainly influence the treatment of male and female patients in the future. From my point of view, in the treatment of oncological patients, gender and hormonal milieu will play an important role in the future. In this respect, hormone receptors have been documented on several gastrointestinal tumors. A trend towards individualizing the treatment (personalized medicine) in those patients is increasingly propagated. Considering gender and hormonal milieu may further improve patient care in this field.

Seeland: Surgery always considers the anatomical differences between the patients as well as their individuality when they are being treated; for example, the use of neoadjuvant and radiation therapy is carefully evaluated in premenopausal women. Nonetheless, the clinical aspects that do not include anatomical differences are generally neglected. Yet, a liver is not just a liver and a stomach is not just a stomach.

Liver function varies by fertility state in women throughout their life course and thus resections, such as lobectomies, might have different effects on metabolism. Also, estrogens seem to play a significant role in the progression of hepatocarcinoma. Survival rates after gastric cancer are significantly better in females compared to males, even after adjustment for size and stage. Nobody knows exactly why this is the case. We know that sex hormones and their receptors play a role but we are far from being able to use this to our advantage when treating our patients.
Question 4: What could be the most effective means of reducing gender-specific surgical site infections (SSI)? Which preventive measures for both sexes are necessary?

Langelotz: The analysis of SSI showed differing results for men and women with respect to the underlying operative procedure. Therefore, there is no straightforward answer to the question as how to reduce the incidence of gender-specific SSI. While there seems to be a hormonal advantage in visceral surgery for women, lowering their incidence of SSI, the opposite effects could be seen in cardiac surgery. Current research suggests a different effect of cardioprotective medication, especially aspirin, on wound healing and SSI in women, leading to a higher rate of SSI. More research is clearly needed on the complex hormonal, cellular, and pharmacological interactions. Until further elucidation of the matter, the general preventive measures for both sexes such as adherence to evidence-based protocols on hand hygiene, preoperative disinfection, atraumatic surgery, and normothermia should be strictly adhered to.

Question 5: Which gender-based adapted screening programs and surgical recommendations can be mentioned regarding hereditary colorectal cancer?

Möslein: The most frequent hereditary colorectal cancer syndromes are Lynch syndrome and familial adenomatous polyposis (FAP), accounting for approximately $5 \%$ of the colorectal cancer burden. Both are characterized by an autosomal dominant mode of transmission and require an individualized approach of intensified screening and prophylactic surgery. Based upon available data, a personalized approach in treating patients and families with these predisposition syndromes is increasingly warranted. In Lynch syndrome patients, men have a significantly higher lifetime risk and earlier age of manifestation for colorectal cancer than females especially in MSH6 mutation carriers. Additionally, incidence of gastric, bladder, and urothelial cancers is much higher in males. Females with an MSH6 mutation have to be aware of a very high risk especially for endometrial, but also for ovarian cancer. In FAP families, females are more prone to papillary thyroid cancers and also to desmoid tumors. I believe that it is timely to suggest gene- and gender-based adapted screening and surgical recommendations for Lynch syndrome patients. Concerning FAP patients, females should follow intensified screening recommendations for early detection of precursors or papillary cancer of the thyroid. Furthermore, desmoid patients should primarily not be subjected to surgical resection but rather to a gender-specific conservative medical treatment. 
Question 6: Which areas should be especially paid heed to in the future? Where are prospective studies most warranted regarding gender-specific differences in order to optimize patient selection and to reduce comorbidities and complications as well as costs?

Kähler: I think that follow-up management, especially how to convince practitioners and patients of the necessity of an individualized follow-up program, would be necessary because this problem is underestimated.

Seeland: Awareness of gender diversity starts at the emergency room and ends with the hospital discharge. What about home care? Is there a recommendation in the hospital discharge letter for clarification of osteoporosis after a fall? Should it be contemplated that men can also be depressive and that the assessment of symptoms, especially pain manifestations, should thus be judged differently? Do gall bladder disease symptoms differ when comparing young and old men and women? How long is the time from the first colic symptoms to intervention? Why do women ask more often for conservative strategies than for surgery? Does it have implications on outcome? Prospective studies could have a look at different procedures comparing both sexes. The identification of areas that should be paid heed to in the future is difficult. The analysis of 'Incident-Reporting Systems' (e.g. CIRS) with regard to sex and gender aspects may help to narrow the field of interest.

From the basic science point of view, the researcher must know the 'sex' of the cells and of the animals used in their studies. The sexes should not be mixed up because they are genetically different, having important implications on epigenetics, metabolomics, and proteomics. For example: Did you know that the patterns of DNA methylation in the normal colon varies by anatomical location, gender, and age? Looking for sex and gender differences in e.g. inflammatory bowel diseases will increase the knowledge about the different phenotypes underlying these diseases.

\section{Question 7: Is there any initiative to address the aspect of gender medicine on a European platform? Why is this necessary?}

Oertelt-Prigione: The recently initiated project EUGenMed (www.eugenmed.eu) aims at exactly this aspect. All over Europe we have regional or national approaches to the inclusion of gender in medicine, oftentimes with best practice examples that are unknown to other researchers in the field. Regulations and resources might differ locally, but many approaches can be adapted - they only need to be shared. To bring all these people together, to discuss the evolution of gender medicine, and to involve stakeholders at all levels of the health care process, the EUGenMed project has been designed. It will feature a series of topic-specific workshops which will lead to a conclusive conference. After the very positive experience of the EUGIM project (http://gender.charite. de/en/education/eugim/), which set out to develop a European curriculum for gender medicine, we are hopeful that it will be possible to extend this experience to other areas.

\section{Participants}

Prof. Dr. med. Martin K. Angele

Klinik für Allgemein-, Viszeral-, Transplantations-, Gefäß- und Thoraxchirurgie

Ludwig-Maximilian-Universität München

Marchioninistraße 15, 81377 München, Germany

Martin.Angele@med.uni-muenchen.de

Dr. Santiago González-Moreno

Department of Surgical Oncology

MD Anderson Cancer Center

C/ Arturo Soria, 270, 28033 Madrid, Spain

sgonzalez@mdanderson.es

Prof. Dr. Georg Kähler

Zentrale Interdisziplinäre Endoskopie ZIE

Universitätsmedizin Mannheim

Theodor-Kutzer-Ufer 1-3, 68167 Mannheim, Germany

Georg.Kaehler@umm.de

Dr. med. Corinna Langelotz

Klinik für Allgemein-, Viszeral-, Gefäß- und Thoraxchirurgie

Charité - Universitätsmedizin Berlin, Campus Mitte

Charitéplatz 1, 10117 Berlin, Germany

corinna.langelotz@charite.de

Prof. Dr. Gabriela Möslein

Abteilung für Allgemeine und Viszeralchirurgie - Koloproktologie HELIOS St. Josefs-Hospital

Axstraße 35, 44879 Bochum, Germany

gabriela.moeslein@helios-kliniken.de

Dr. Sabine Oertelt-Prigione, MScPH

Institut für Geschlechterforschung in der Medizin (GiM)

Charité - Universitätsmedizin Berlin, Campus Mitte

Luisenstraße 65, 10117 Berlin, Germany

sabine.oertelt-prigione@charite.de

PD Dr. med. Andrea Riphaus

Medizinische Klinik II - Gastroenterologie

KRH Klinikum Agnes Karll

Hildesheimer Straße 158, 30880 Laatzen, Germany

Andrea.Riphaus@rub.de

Dr. med. Ute Seeland

Institut für Geschlechterforschung in der Medizin (GiM)

Charité - Universitätsmedizin Berlin, Campus Mitte

Luisenstraße 65, 10117 Berlin, Germany

ute.seeland@charite.de 